\title{
NbN superconducting nanowire photon counters: magnetoconductivity and other detector properties
}

\author{
Engel, A ; Bartolf, H ; Gómez, L ; Semenov, A ; Il’in, K ; Schilling, A ; Hübers, H W ; Siegel, M
}

\begin{abstract}
We present an analysis of magnetoconductivity measurements on $\mathrm{NbN}$ thin films and nanostructures as they are used for superconducting nanowire single-photon detectors. Especially the temperaturedependence of the fluctuation conductivity near the zero-field transition temperature was studied in detail. Together with complementary measurements of the optical constants of $\mathrm{NbN}$ films with varying thickness, strategies for improving the absorptivity in $\mathrm{NbN}$ thin films are developed. $\odot 2009$ American Institute of Physics
\end{abstract}

DOI: https://doi.org/10.1063/1.3292452

Posted at the Zurich Open Repository and Archive, University of Zurich

ZORA URL: https://doi.org/10.5167/uzh-27269

Journal Article

Published Version

Originally published at:

Engel, A; Bartolf, H; Gómez, L; Semenov, A; Il'in, K; Schilling, A; Hübers, H W; Siegel, M (2009). NbN superconducting nanowire photon counters: magnetoconductivity and other detector properties. AIP Conference Proceedings, 1185:76-79.

DOI: https://doi.org/10.1063/1.3292452 


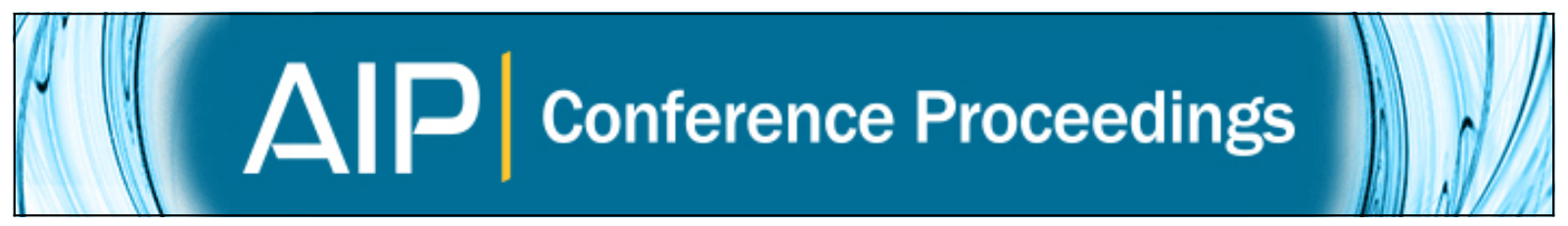

$\mathrm{NbN}$ superconducting nanowire photon counters: magnetoconductivity and other detector properties

Andreas Engel, Holger Bartolf, Luis Gómez, Alexei Semenov, Konstantin Il'in, Andreas Schilling, Heinz-Wilhelm Hübers, and Michael Siegel

Citation: AIP Conference Proceedings 1185, 76 (2009); doi: 10.1063/1.3292452

View online: http://dx.doi.org/10.1063/1.3292452

View Table of Contents: http://scitation.aip.org/content/aip/proceeding/aipcp/1185?ver=pdfcov

Published by the AIP Publishing

Articles you may be interested in

Dependence of Excess Noise on the Partial Derivatives of Resistance in Superconducting Transition Edge Sensors

AIP Conf. Proc. 1185, 31 (2009); 10.1063/1.3292343

Microwave Kinetic Inductance Detectors: The First Decade

AIP Conf. Proc. 1185, 135 (2009); 10.1063/1.3292300

Multi-channeled $\mathrm{NbN}$ superconducting single photon detectors (SSPDs) system with $\mathrm{NbN}$ meander nanowires AIP Conf. Proc. 1110, 279 (2009); 10.1063/1.3131327

IF impedance and mixer gain of $\mathrm{NbN}$ hot electron bolometers

J. Appl. Phys. 101, 044511 (2007); 10.1063/1.2400086

APL Photonics 


\title{
NbN superconducting nanowire photon counters: magnetoconductivity and other detector properties
}

\author{
Andreas Engel*, Holger Bartolf*, Luis Gómez*, Alexei Semenov ${ }^{\dagger}, K^{*}$ onstantin \\ Il'in**, Andreas Schilling*, Heinz-Wilhelm Hübers ${ }^{\dagger}$ and Michael Siegel** \\ ${ }^{*}$ Physics Institute, University of Zurich, Switzerland \\ ${ }^{\dagger} D L R$ Institute of Planetary Research, Berlin, Germany \\ ${ }^{* *}$ Institute of Micro- und Nano-electronic Systems, University of Karlsruhe, Germany
}

\begin{abstract}
We present an analysis of magnetoconductivity measurements on $\mathrm{NbN}$ thin films and nanostructures as they are used for superconducting nanowire single-photon detectors. Especially the temperature-dependence of the fluctuation conductivity near the zero-field transition temperature was studied in detail. Together with complementary measurements of the optical constants of $\mathrm{NbN}$ films with varying thickness, strategies for improving the absorptivity in NbN thin films are developed.
\end{abstract}

Keywords: fluctuation conductivity, single-photon detector, niobium nitride, thin film PACS: 74.78. $\mathrm{Db}, 74.78 . \mathrm{Na}, 85.25 . \mathrm{Pb}$

\section{INTRODUCTION}

Recent progress in the research on superconducting nanowire single-photon detectors (SNSPD) made from NbN ultrathin films make them very interesting detectors in particular for communication applications, such as quantum key distribution. It has been realized that the kinetic inductance of the superconducting meander is the key parameter limiting their speed [1]. Due to the large effective penetration depth and high aspect ratio, the kinetic inductance can reach very large values for large area detectors. In order to achieve large area detectors, but at the same time retain the fast response characteristic for these detectors, various segmented or parallel wire configurations have been proposed [2,3]. These new geometries have the added advantage that they, in principle, allow the realization of photon-number resolving detectors [4].

However, a finite value of the kinetic inductance is important for a stable operation of the detectors [5]. If the kinetic inductance is reduced too much in an attempt to increase the detector's speed, the phenomenon of latching into the normal conducting state prohibits the intrinsic recovery of superconductivity after the absorption of a photon.

Insofar as the speed limit of SNSPDs is concerned a consistent picture has emerged. But some other issues remain to be addressed to realize the full potential of these detectors. One problem is the limited quantum efficiency and the need for a better understanding of the parameters influencing this quantity. Despite all the progress in fabrication technologies it is still puzzling that reported maximum quantum efficiencies scatter over at least one order of magnitude for otherwise comparable detectors $[6,7,8,9,10]$.

This observation maybe a manifestation of the fact that the overall quantum efficiency is determined by many different factors: optical properties, the coupling of the incoming electromagnetic wave to the nanostructure and the intrinsic detection mechanism. The coupling of the radiation to the nanostructure depends on details of the layout and geometry with respect to the wavelength to be detected and can be significantly improved by integrating the SNSPD into an optical cavity [11], for example. In this paper we will focus on the electronic and superconducting properties of $\mathrm{NbN}$ thin films and nanostructures. A careful analysis of magnetoconductivity data allows us a comprehensive understanding of the superconducting state and the determination of relevant microscopic material parameters. Relating these results to the optical properties of $\mathrm{NbN}$ thin films we obtain a better understanding of the factors influencing the absorptivity and quantum efficiency of SNSPD devices.

\section{EXPERIMENTAL DETAILS}

The superconducting $\mathrm{NbN}$ thin films were prepared by $\mathrm{DC}$ magnetron sputtering in an $\mathrm{Ar}+\mathrm{N}_{2}$ atmosphere. Films were deposited onto polished sapphire substrates. Growth conditions, such as nitrogen partial pressure, substrate temperature and growth rate, were optimized to achieve maximum critical temperature $T_{c}$ for the resulting films, see Ref. [12] for details. Using standard photo- and electron-beam lithography and ion milling newly deposited films were then structured into 

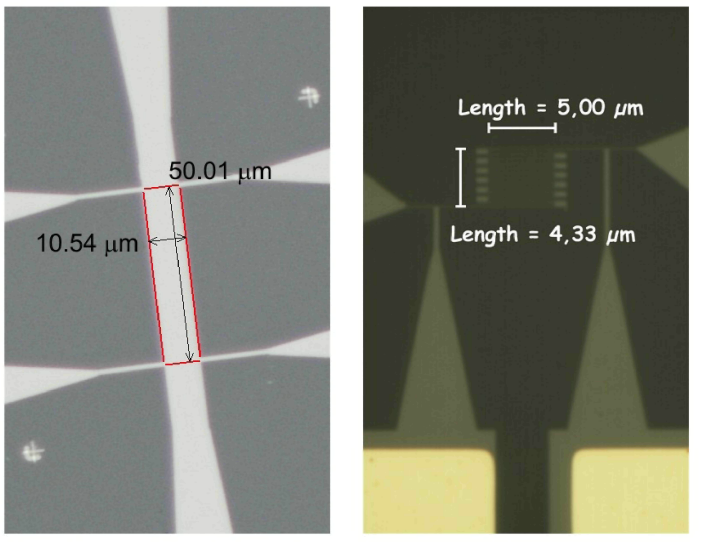

FIGURE 1. Optical microscope pictures of a NbN bridge (left) and meander (right).

TABLE 1. Geometric parameters (thickness $d$, width $w$ and length $L$ ) of the samples. B indicates a simple bridge, $\mathrm{M}$ stands for a detector meander.

\begin{tabular}{lccc}
\hline & $d(\mathbf{n m})$ & $w(\mu \mathbf{m})$ & $L(\mu \mathbf{m})$ \\
\hline B3 & 3.2 & 10.1 & 50 \\
B6 & 6.0 & 10.4 & 50 \\
B12 & 12 & 10.5 & 50 \\
M50 & 6.0 & 0.053 & 74 \\
\hline
\end{tabular}

micrometer-sized bridges for four-point conductivity and hall-effect measurements. Sub-micrometer-sized meander structures were fabricated by a slightly different lithography process using plasma-etching in a $\mathrm{SF}_{6} / \mathrm{Ar}$ atmosphere [13]. In Fig. 1 we show optical microscope pictures of a bridge and a meander. The sub-micrometer linewidths of the meanders were determined from scanning electron microscope images.

Magnetoconductivity measurements were done in a commercial cryostat (Physical Properties Measurement System, Quantum Design) equipped with a 9 T superconducting solenoid. The resistance measurements were done with excitation currents at least two orders of magnitude lower than typical critical currents in the superconducting state.

\section{ANALYSIS OF CONDUCTIVITY MEASUREMENTS}

In Fig. 2 magnetoconductivity data near $T_{c}(H)$ are shown for sample B12 for magnetic inductions up to $4 \mathrm{~T}$. Using the midpoint of the transition at $R(T)=R_{n} / 2$ as the critical temperature at the applied magnetic field, we obtain the upper critical field line in the $H-T$ plane (red line in the inset of Fig. 2). It is to a good approximation lin-

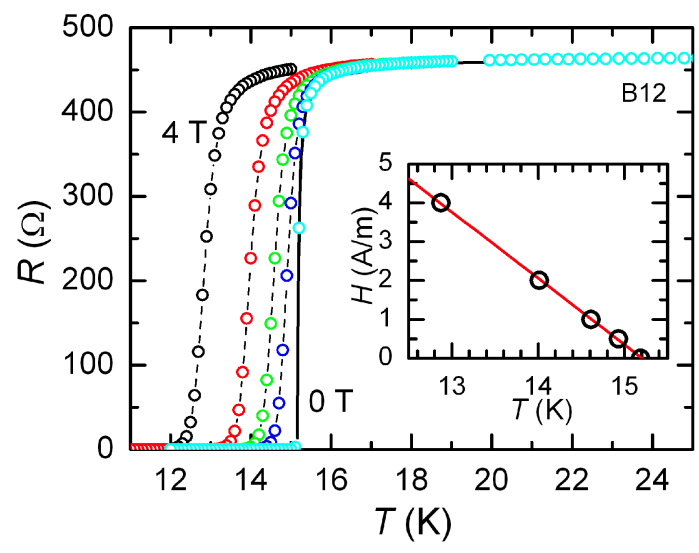

FIGURE 2. Resistance vs. temperature for sample B12 in fields of $0,0.5,1,2$ and $4 \mathrm{~T}$ (right to left). Dashed lines are guidelines only, the thick solid line is a least-square fit of Eq. 2 with $m=2$ to the zero-field data. The inset shows $H_{c 2}(T)$ and a linear fit to the data points (red line).

TABLE 2. Parameters of the superconducting and the normal-conducting states as derived from our magnetoconductivity measurements (see text).

\begin{tabular}{lcccc}
\hline & $T_{c}(\mathbf{K})$ & $\xi_{0}(\mathbf{n m})$ & $\rho_{n}(\mu \Omega \mathbf{m})$ & $D\left(\mathbf{c m}^{2} / \mathbf{s}\right)$ \\
\hline B3 & 10.7 & 3.7 & 3.0 & 0.52 \\
B6 & 14.1 & 3.6 & 1.5 & 0.62 \\
B12 & 15.2 & 3.6 & 1.2 & 0.64 \\
M50 & 12.4 & 3.4 & 2.4 & 0.49 \\
\hline
\end{tabular}

ear in $T$ as predicted in Ginzburg-Landau (GL) theory [14]. From a linear least-squares fit we obtain the extrapolated $H_{c 2}(0)$, from which we can determine the wellknown GL-coherence length $\xi_{0}^{2}=\Phi_{0} /\left(2 \pi \mu_{0} H_{c 2}(0)\right)$, with $\Phi_{0}=h / 2 e$ the magnetic flux quantum, $e$ the elementary charge and $\mu_{0}$ the permittivity of free space. It is less known that it is possible to extract the diffusion constant of the normal electrons from the slope of the upper critical field at $T_{c}=T_{c}(0)$ [15]

$$
D=-\frac{4 k_{B}}{\left.\pi e \mu_{0} \frac{\mathrm{d} H_{c 2}}{\mathrm{~d} T}\right|_{T=T_{C}},}
$$

with $k_{B}$ being the Boltzmann-constant. Numerical values are given in table 2 .

Except for sample B12 one expects the structures to be predominantly two-dimensional in character, because $\xi(T) \geq d$ for at least a significant range of temperatures around $T_{c}$. In order to verify this assumption we tried to describe the resistance data above $T_{c}$ by making use of the fluctuation conductivity theory of Aslamazov and Larkin [16]. Expressed in terms of the measured resistance $R$ and arbitrary dimensionality $m=1,2,3$ it renders 
to

$$
R(T)=\frac{R_{n}}{1+R_{n} A_{m}\left(\frac{T}{T_{c}}-1\right)^{2-0.5 m}}, \quad\left(T>T_{c}\right),
$$

with $R_{n}$ the normal state resistance and $A_{m}$ a constant factor depending on the dimensionality [17]. We tried to fit the $R(T)$-data in a small temperature range $T_{c}^{\text {est }}<$ $T<1.1 T_{c}^{\text {est }}$, with $T_{c}^{\text {est }}$ the estimated $T_{c}$ from the $R_{n} / 2$ criterion, to Eq. (2) using all three values for $m$. Indeed, for samples B3 and B6 only the 2D-variant of Eq. 2 results in a good fit to the experimental data. The quality of these best fits is probably best demonstrated by extrapolating them to $T=25 \mathrm{~K}$, far beyond the fitting range. In this way extrapolated expectation values deviate by less than $1 \%$ from experimental data. This seems to be a systematic deviation that is possibly caused by our simplification of a temperature independent normal state resistance. The $T_{c}$ and $\rho_{n}$ values in Tab. 2 were determined from these fits. Film thicknesses $d$ inferred from the prefactor $A_{2}=e^{2} /(16 \hbar d)$ are only a factor 2 smaller than the thicknesses given in Tab. 1 determined from the sputter rate and the sputter time or atomic force microscopy measurements of $d$.

The situation is somewhat more complicated for the other two samples. Using the same $10 \%$ temperature range above $T_{c}$ the resistance of B12 can be well fitted with the 2D-equation of the fluctuation conductivity (red curve in Fig. 3). From the fit parameters we obtained $T_{c}, \rho_{n}$ (Tab. 2) and an apparent thickness of $d \approx 8 \mathrm{~nm}$. Also shown is a fit with $m=3$, which clearly does not describe the experimental data near $T_{c}$, and a similarly clear deviation from the data is observed for a fit with $m=1$ (not shown in the graph).

However, an extrapolation to higher temperatures fails even if one extends the fitted temperature range to $25 \mathrm{~K}$ (inset of Fig. 3). Depending on the used temperature range, the $2 \mathrm{D}$-fit deviates systematically from the data starting at temperatures between about 17 and $18 \mathrm{~K}$. But the 3D-equation of the fluctuation conductivity describes the data very well in this temperature range (blue curve). Using the appropriate pre-factor $A_{3}=5 e^{2} /\left(32 \hbar \xi_{0}\right)$, including the Maki-Thompson correction [18, 19], the coherence length computes to $3.9 \mathrm{~nm}$, in very good agreement with our other measurements. Using the GLcoherence length $\xi_{0}$ obtained from the magnetoconductivity measurements, the corresponding length-scale above $T_{c}$ drops from about 10 to $8 \mathrm{~nm}$ between 17 and $18 \mathrm{~K}$ indicating a $2 \mathrm{D}-3 \mathrm{D}$ cross-over in this temperature range.

At the other end of the scale is the meander structure with a cross-sectional area of about $S=318 \mathrm{~nm}^{2}$. Neglecting the high aspect-ratio of the conduction path, the condition for one-dimensional behavior, $\xi(T)^{2} \geq S$, is fulfilled in a narrow temperature interval of about $5 \%$

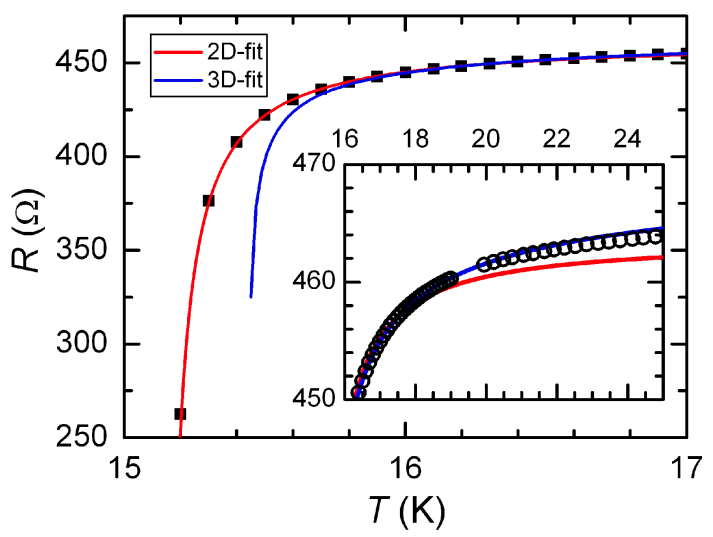

FIGURE 3. Resistance vs. temperature for sample B12 in zero-field, with least-square fits of Eq. 2 with $m=2$ (red line) and $m=3$ (blue line) in a $10 \%$ interval above $T_{c}$. The inset shows the fits at higher temperatures, where the 3D-equation appears to better describe the data. Around $18 \mathrm{~K}$ the $2 \mathrm{D}$-fit deviates from the temperature-dependence of the experimental data.

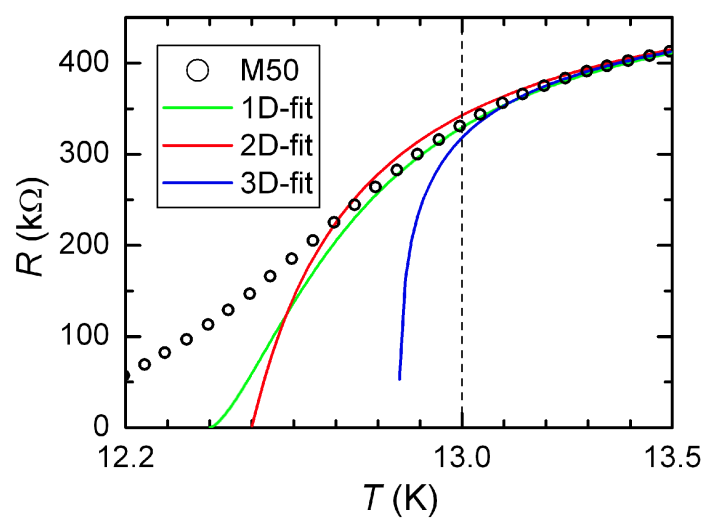

FIGURE 4. Resistance vs. temperature in zero-field for sample M50 and three least-square fits with $m=1,2$ and 3. Qualitatively and quantitatively, the $1 \mathrm{D}$-equation fits the data best. The vertical dashed line indicates the expected cross-over from 1 to $2 \mathrm{D}$, solely based on the cross-sectional area and the GL temperature-dependence of $\xi$.

around $T_{c}$. Based on this argument we expect a 1D-2D cross-over around $13 \mathrm{~K}$. Whereas only the fit with $m=2$ gives satisfactory results at higher temperatures, again with a seamless extrapolation to higher temperatures, the fit with $m=1$ describes the experimental data better very close to $T_{c}$, see also Fig. 4 . In particular the curvature of 1D-equation matches the experimental data well around $13 \mathrm{~K}$, whereas the $2 \mathrm{D}$-fit shows small but systematic deviations. Nevertheless, in the case of this narrow meander line we cannot exclude the possibility of a broadened transition due to larger relative line-width variations. 


\section{CONCLUSIONS}

From the analysis of the fluctuation conductivity above $T_{C}$ one can obtain a clear picture about the effective dimensionality of $\mathrm{NbN}$ thin-film structures. Taking into account the temperature dependence of the smallest relevant length scale in this problem, the GL-coherence length $\xi(T)$, these structures undergo dimensional crossovers as expected according to their geometry. With respect to NbN SNSPD we can draw the conclusion that for most meander structures examined so far the assumption of two-dimensionality was justified. Even conduction paths as narrow as $50 \mathrm{~nm}$ show signs of onedimensional behavior in a very small temperature interval around $T_{C}$ only. For typical operation temperatures $T<T_{C} / 2$, the condition $\xi(T) \ll w$ brings them well into a two-dimensional regime.

In order to increase the overall detection efficiency one possible approach would be to increase the film thickness. In a complementary study [20] we have determined the optical properties of $\mathrm{NbN}$ thin films with different thicknesses using ellipsometry. From those results we expect a maximum in the absorptivity in the near-infrared for $\mathrm{NbN}$ thin films with a thickness of around $8 \mathrm{~nm}$. Although our analysis suggests that even $8 \mathrm{~nm}$ thick films can be well described with two-dimensional models, detection models predict a linear increase of the required cut-off photon energy with increasing film thickness [21], which counteracts the increase in absorptivity. Due to the near exponential decrease in the quantum efficiency for photon energies smaller than the cut-off energy the overall quantum efficiency in the near-infrared is usually even reduced with an increase in film thickness.

Based on the experimentally determined optical constants of $\mathrm{NbN}$ thin films, one expects monotonically increasing absorptivity with increasing film thickness for the visible range and higher photon energies. For applications in this spectral range one might be able to increase the quantum efficiency using thicker films, as long as the cut-off energy remains smaller than the photon energy and the two-dimensional detection model remains valid. The latter condition should be fulfilled at least up to about $10 \mathrm{~nm}$ film thickness.

\section{ACKNOWLEDGMENTS}

H. B. acknowledges support by the Swiss NCCR Manep.

\section{REFERENCES}

1. A. J. Kerman, E. A. Dauler, W. E. Keicher, J. K. W. Yang, K. K. Berggren, G. Gol'tsman, and B. Voronov, Appl. Phys. Lett. 88, 111116 (2006).
2. M. Ejrnaes, R. Cristiano, O. Quaranta, S. Pagano, A. Gaggero, F. Mattioli, R. Leoni, B. Voronov, and G. Gol'tsman, Appl. Phys. Lett. 91, 262509 (2007).

3. A. Korneev, A. Divochiy, M. Tarkhov, O. Minaeva, V. Seleznev, N. Kaurova, B. Voronov, O. Okunev, G. Chulkova, I. Milostnaya, K. Smirnov, and G. Gol'tsman, J. Phys.: Condens.Matter 97, 012307 (2008).

4. A. Divochiy, F. Marsili, D. Bitauld, A. Gaggero, R. Leoni, F. Mattioli, A. Korneev, V. Seleznev, N. Kaurova, O. Minaeva, G. Gol'tsman, K. G. Lagoudakis, M. Benkhaoul, F. Lévy, and A. Fiore, Nature Photonics 2, 302 (2008).

5. A. J. Kerman, J. K. W. Yang, R. J. Molnar, E. A. Dauler, and K. K. Berggren, Phys. Rev. B 79, 100509(R) (2009).

6. G. N. Gol'tsman, A. Korneev, I. Rubtsova, I. Milostnaya, G. Chulkova, O. Minaeva, K. Smirnov, B. Voronov, W. Slysz, A. Pearlman, A. Verevkin, and R. Sobolewski, phys. stat. sol. (c) 2, 1480 (2005).

7. A. Korneev, V. Matvienko, O. Minaeva, I. Milostnaya, I. Rubtsova, G. Chulkova, K. Smirnov, V. Voronov, G. Gol'tsman, W. Slysz, A. Pearlman, A. Verevkin, and R. Sobolewski, IEEE Trans. Appl. Supercon. 15, 571 (2005).

8. A. J. Kerman, E. A. Dauler, J. K. W. Yang, K. M. Rosfjord, V. Anant, K. K. Berggren, G. Gol'tsman, and B. Voronov, Appl. Phys. Lett. 90, 101110 (2007).

9. R. Leoni, A. Gaggero, F. Mattioli, M. G. Castellano, P. Carelli, F. Marsili, D. Bitauld, M. Benkahoul, F. Lévy, and A. Fiore, J. Low Temp. Phys. 151, 580 (2008).

10. S. Miki, M. Fujiwara, M. Sasaki, B. Baek, A. J. Miller, R. H. Hadfield, S. W. Nam, and Z. Wang, Appl. Phys. Lett. 92, 061116 (2008).

11. V. Anant, A. J. Kerman, E. A. Dauler, J. K. W. Yang, K. M. Rosfjord, and K. K. Berggren, Opt. Express 16, 10750 (2008).

12. K. Il'in, M. Siegel, A. Engel, H. Bartolf, A. Schilling, A. Semenov, and H.-W. Hübers, J. Low Temp. Phys. 151, 585-590 (2008).

13. H. Bartolf, A. Engel, A. Schilling, K. Il'in, and M. Siegel, Phys. C 468, 793 (2008).

14. V. L. Ginzburg, and L. D. Landau, Zh. Eksperim. Teor. Fiz. 20, 1064 (1950).

15. D. Saint-James, G. Sarma, and E. J. Thomas, Type II superconductivity, Pergamon Press, 1969.

16. L. G. Aslamazov, and A. I. Larkin, Phys. Lett. 26A, 238 (1968).

17. M. Tinkham, Introduction to Superconductivity, McGrawHill, Inc., New York, 1996, second edn.

18. K. Maki, Prog. Theor. Phys. 40, 193-200 (1968).

19. R. S. Thompson, Phys. Rev. B 1, 327-333 (1970).

20. A. Semenov, P. Haas, H.-W. Hübers, K. Ilin, M. Siegel, A. Kirste, D. Drung, T. Schurig, and A. Engel, J. Mod. Optics 56, 345 (2009).

21. A. Semenov, A. Engel, H.-W. Hübers, K. Il'in, and M. Siegel, Eur. Phys. J. B 47, 495 (2005). 